PROCEEDINGS OF THE

AMERICAN MATHEMATICAL SOCIETY

Volume 126, Number 12, December 1998, Pages 3657-3660

S 0002-9939(98)04589-4

\title{
A CHARACTERIZATION OF ROUND SPHERES
}

\author{
SUNG-EUN KOH
}

(Communicated by Christopher Croke)

\begin{abstract}
A new characterization of geodesic spheres in the simply connected space forms in terms of higher order mean curvatures is given: An immersion of an $n$ dimensional compact oriented manifold without boundary into $n+1$ dimensional Euclidean space, hyperbolic space or the open half sphere is a totally umbilic immersion if, for some $r, r=2, \ldots, n$, the $(r-1)$-th mean curvature $H_{r-1}$ does not vanish and the ratio $H_{r} / H_{r-1}$ is constant.
\end{abstract}

\section{INTRODUCTION}

Let $N^{n+1}$ be one of the Euclidean space $\mathbf{R}^{n+1}$, the hyperbolic space $\mathbf{H}^{n+1}$ or the open half sphere $\mathbf{S}_{+}^{n+1}$, and let $\phi: M^{n} \rightarrow N^{n+1}$ be an isometric immersion of a compact oriented $n$ dimensional manifold without boundary $M^{n}$. Let $H_{r}$ denote the $r$-th mean curvature of $M^{n}$, that is, $H_{r}$ is the $r$-th elementary symmetric polynomial of principal curvatures of $M^{n}$ divided by $\left(\begin{array}{l}n \\ r\end{array}\right)$, and $H_{0}$ is defined to be 1. For instance, $H_{1}$ is the usual mean curvature and $H_{n}$ is the Gauss-Kronecker curvature.

It was shown in [3] that if two consecutive mean curvatures $H_{r-1}, H_{r}$ are constant for some $r$ between 2 and $n$, then $\phi\left(M^{n}\right)$ is a geodesic sphere. In this note, we generalize this result in the following way.

Theorem. Let $N^{n+1}$ be one of $\mathbf{R}^{n+1}, \mathbf{H}^{n+1}$ or $\mathbf{S}_{+}^{n+1}$, and let $\phi: M^{n} \rightarrow N^{n+1}$ be an isometric immersion of a compact oriented n-dimensional manifold without boundary $M^{n}$. If $H_{r-1}$ does not vanish and the ratio $H_{r} / H_{r-1}$ of two consecutive mean curvatures is a constant for some $r=2, \ldots, n$, then $\phi\left(M^{n}\right)$ is a geodesic hypersphere.

When $N^{n+1}$ is $\mathbf{R}^{3}$, the above theorem was proved in [1] under the convexity assumption. Note that we consider immersions, and the case $r=1$ is omitted. As we defined $H_{0}$ to be 1 , the ratio $H_{1} / H_{0}$ is equal to the usual mean curvature $H_{1}$. Because of the existence of nonspherical immersions of nonzero constant mean curvature $H_{1}$ in $\mathbf{R}^{n}$, proved by Hsiang, Teng and $\mathrm{Yu}$ [4] and Wente [6], we cannot expect our theorem for $r=1$. However, by Alexandrov's well-known sphere theorem

Received by the editors April 25, 1997.

1991 Mathematics Subject Classification. Primary 53C40, 53C42.

Key words and phrases. Higher order mean curvature, principal curvature, umbilical point, Minkowski formula.

This research was supported by the KOSEF through Research Fund 96-0701-02-01-3, and by the Korean Ministry of Education through Research Fund BSRI-96-1438.

(C)1998 American Mathematical Society 
or Montiel and Ros' theorem [5], our theorem holds even for $r=1$ if we assume embeddings.

\section{ProOF}

We use the hyperboloid model for $\mathbf{H}^{n+1}$ and the usual embedding of $\mathbf{S}^{n+1}$ into $\mathbf{R}^{n+2}$. Let $\eta$ denote a unit normal field on $M^{n}$. We use the following Minkowski formula (for proof, see [5]), where $\langle$,$\rangle denotes the usual Euclidean inner product$ on $\mathbf{R}^{n+1}$ (on $\mathbf{R}^{n+2}$ ) when $N^{n+1}$ is $\mathbf{R}^{n+1}$ (when $N^{n+1}$ is $\mathbf{S}_{+}^{n+1}$ ) and the Lorentzian inner product on $\mathbf{R}^{n+2}$ when $N^{n+1}$ is $\mathbf{H}^{n+1}$.

Lemma 1. For $k=1, \ldots, n$, the following identities hold:

(1) When $N^{n+1}$ is $\mathbf{R}^{n+1}$,

$$
\int_{M}\left(H_{k-1}+H_{k}\langle\phi, \eta\rangle\right) d M=0 .
$$

(2) When $N^{n+1}$ is $\mathbf{H}^{n+1}$,

$$
\int_{M}\left(H_{k-1}\langle\phi, p\rangle+H_{k}\langle\eta, p\rangle\right) d M=0 \text { for any } p \in \mathbf{R}^{n+2} \text {. }
$$

(3) When $N^{n+1}$ is $\mathbf{S}_{+}^{n+1}$,

$$
\int_{M}\left(H_{k-1}\langle\phi, p\rangle-H_{k}\langle\eta, p\rangle\right) d M=0 \text { for any } p \in \mathbf{R}^{n+2} \text {. }
$$

We also use the following inequalities for higher order mean curvatures (for proof, see, for example, $\S 12$ of [2]):

Lemma 2. (1) $H_{r} H_{r-2} \leq H_{r-1}^{2}$.

(2) If $H_{1}, H_{2}$ are greater than zero everywhere on $M^{n}$, then

$$
H_{2} \leq H_{1}{ }^{2}
$$

and the equality holds only at umbilical points.

Now, assume $H_{r-1}$ does not vanish and $H_{r} / H_{r-1}=a$ for a constant number $a$ and a fixed $r=2, \ldots, n$.

(2.1) Proof when $N^{n+1}=\mathbf{R}^{n+1}$. Since $M^{n}$ is compact, by changing the unit normal vector on $M$ if necessary, one can find a point in $M^{n}$ where all the principal curvatures are positive. Then $H_{r}, H_{r-1}$ are positive at that point. Since $H_{r-1}$ does not vanish and $H_{r} / H_{r-1}$ is constant on $M^{n}, H_{r}$ is positive on $M^{n}$. Then, following the argument of Lemma 1 in [5], it follows that the $H_{k}$ are positive on $M^{n}$ for $k=1, \ldots, r-1$. Then $a>0$ and from the inequality (1)

$$
H_{r} H_{r-2} \leq H_{r-1}^{2}
$$

of Lemma 2, we have

$$
0<a=H_{r} / H_{r-1} \leq H_{r-1} / H_{r-2} .
$$

Since $H_{r}=a H_{r-1}$, we have by Lemma 1 ,

$$
\begin{aligned}
0 & =\int_{M}\left(H_{r-1}+H_{r}\langle\phi, \eta\rangle\right) d M \\
& =\int_{M}\left(H_{r-1}+a H_{r-1}\langle\phi, \eta\rangle\right) d M,
\end{aligned}
$$


that is,

$$
\int_{M} H_{r-1}\langle\phi, \eta\rangle d M=\int_{M}\left(-\frac{1}{a} H_{r-1}\right) d M .
$$

Since

$$
\int_{M}\left(H_{r-2}+H_{r-1}\langle\phi, \eta\rangle\right) d M=0
$$

we then have

$$
\int_{M}\left(H_{r-2}-\frac{1}{a} H_{r-1}\right) d M=0 .
$$

Since $H_{r-2}-\frac{1}{a} H_{r-1} \leq 0$ from $\left(^{*}\right)$, it follows that

$$
H_{r-1} / H_{r-2}=a=H_{r} / H_{r-1},
$$

everywhere on $M^{n}$. Now, proceeding inductively, we have finally

$$
H_{2} / H_{1}=a=H_{1} / H_{0}=H_{1}
$$

everywhere on $M^{n}$, that is, the equality holds in (2) of Lemma 2. Hence every point is an umbilical point, i.e., $\phi\left(M^{n}\right)$ is a geodesic hypersphere.

(2.2) Proof when $N^{n+1}=\mathbf{H}^{n+1}$. At a point of $M^{n}$ where the distance function of $\mathbf{H}^{n+1}$ attains its maximum, all the principal curvatures are positive. Then $\left(^{*}\right)$ also holds in this case, and the $H_{k}$ are positive on $M^{n}$ for $k=1, \ldots, r-1$. Since $H_{r}=a H_{r-1}$, we have

$$
\begin{aligned}
0 & =\int_{M}\left(H_{r-1}\langle\phi, p\rangle+H_{r}\langle\eta, p\rangle\right) d M \\
& =\int_{M}\left(H_{r-1}\langle\phi, p\rangle+a H_{r-1}\langle\eta, p\rangle\right) d M
\end{aligned}
$$

that is,

$$
\int_{M} H_{r-1}\langle\eta, p\rangle d M=\int_{M}\left(-\frac{1}{a} H_{r-1}\langle\phi, p\rangle\right) d M .
$$

Since

$$
\int_{M}\left(H_{r-2}\langle\phi, p\rangle+H_{r-1}\langle\eta, p\rangle\right) d M=0,
$$

it follows that

$$
\int_{M}\left(H_{r-2}-\frac{1}{a} H_{r-1}\right)\langle\phi, p\rangle d M=0 .
$$

Now, if we take $p=(1,0, \ldots, 0) \in \mathbf{R}^{n+2}$, then the sign of $\langle\phi, p\rangle$ does not change on $M^{n}$. Since $H_{r-2}-\frac{1}{a} H_{r-1} \leq 0$ from $\left(^{*}\right)$, we have $H_{r-2}-\frac{1}{a} H_{r-1}=0$ everywhere on $M^{n}$. Proceeding inductively as in (2.1), we can show that every point is an umbilical point. Hence $\phi\left(M^{n}\right)$ is a geodesic hypersphere. 
(2.3) Proof when $N^{n+1}=\mathbf{S}_{+}^{n+1}$. Let $c \in \mathbf{S}^{n+1}$ be the centre of $\mathbf{S}_{+}^{n+1}$. Then at a point of $M^{n}$ where the height function $\langle\phi, c\rangle$ attains its maximum, all the principal curvatures are positive because $M^{n}$ lies in the open half sphere with the centre $c$. Then $(*)$ holds, and the equality in $(*)$ holds only at umbilical points. Proceeding as in (2.2), we have

$$
\int_{M}\left(H_{r-2}-\frac{1}{a} H_{r-1}\right)\langle\phi, p\rangle d M=0 .
$$

Since $M^{n}$ lies in the open half sphere, one can find a vector $p \in \mathbf{R}^{n+2}$ so that $\langle\phi, p\rangle$ is positive on $M^{n}$. Then, since $H_{r-2}-\frac{1}{a} H_{r-1} \leq 0$ by $\left(^{*}\right)$, it follows that $H_{r-2}-\frac{1}{a} H_{r-1}=0$, everywhere on $M^{n}$. Now, arguing in the same way as above, we can show that $\phi\left(M^{n}\right)$ is a geodesic hypersphere.

\section{REFERENCES}

1. K. Amur, On a characterization of the 2-sphere, American Mathematical Monthly 78 (1971), 382-384. MR 43:5463

2. E. F. Beckenbach, R. Bellman, Inequalities, Springer Verlag, Berlin, 1971. MR 33:236 (earlier ed.)

3. I. Bivens, Integral formulas and hyperspheres in a simply connected space form, Proc. Amer. Math. Soc. 88 (1983), 113-118. MR 84k:53052

4. W. Y. Hsiang, Z. H. Teng, W.C. Yu, New examples of constant mean curvature immersions of (2k-1)-spheres into Euclidean 2k-space, Ann. of Math. 117 (1983), 609-625. MR 84i:53057

5. S. Montiel, A. Ros, Compact hypersurfaces: The Alexandrov theorem for higher order mean curvatures, Differential Geometry (B. Lawson, ed.), Pitman Mono. 52, Longman, New York, 1991, pp. 279-296. MR 93h:53062

6. H. C. Wente, Counterexample to a conjecture of H. Hopf, Pacific J. Math. 121 (1986), 193-243. MR 87d:53013

Department of Mathematics, Kon-Kuk University, Seoul, 143-701, Korea

E-mail address: sekoh@kkucc.konkuk.ac.kr 